
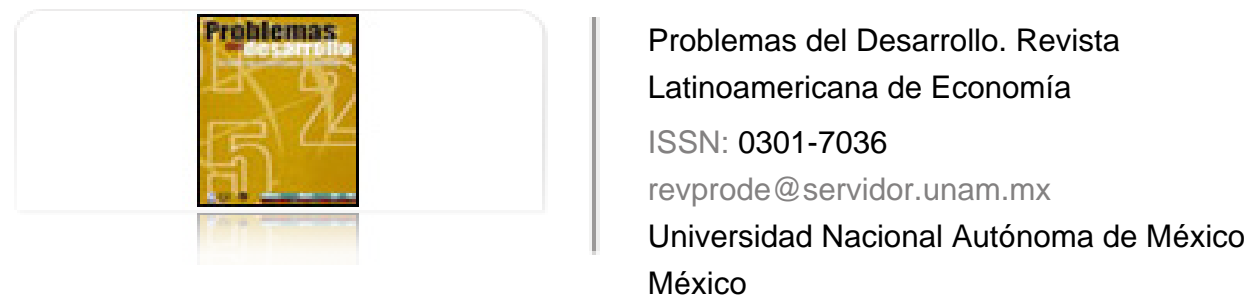

Rivera Ríos, Miguel Ángel

Cambio histórico mundial, capitalismo informático y economía del conocimiento Problemas del Desarrollo. Revista Latinoamericana de Economía, vol. 36, núm. 141, abril-junio, 2005, pp. 27-58

Universidad Nacional Autónoma de México

Distrito Federal, México

Disponible en: http://www.redalyc.org/articulo.oa?id=11820075003

- Cómo citar el artículo

- Número completo

- Más información del artículo

Página de la revista en redalyc.org

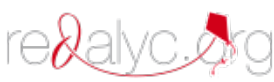

Sistema de Información Científica

Red de Revistas Científicas de América Latina, el Caribe, España y Portugal Proyecto académico sin fines de lucro, desarrollado bajo la iniciativa de acceso abierto 


\section{CAMBIO HISTÓRICO MUNDIAL, CAPITALISMO INFORMÁTICO Y ECONOMÍA DEL CONOCIMIENTO*}

\section{Miguel Ángel Rivera Ríos*}

Fecha de recepción: 16 de diciembre de 2004. Fecha de aceptación: 15 de junio de 2005.

\section{Resumen}

El artículo aborda la discusión acerca del cambio histórico del capitalismo y el papel que juega en ese proceso la emergencia de la llamada economía del conocimiento. Se formula un marco teórico e histórico y se proponen varias hipótesis cuya integración conduce a caracterizar la especificidad de la etapa actual del capitalismo. La economía del conocimiento se instaura sobre transformaciones epistemológicas en la tecnología y en una reducción dramática de los costos sociales de acceso al conocimiento útil. Las rentas tecnológicas que emanan de la difusión del nuevo paradigma y de la constitución de su patrón industrial hacen necesaria la aparición de un nuevo régimen que posibilite reordenar las relaciones de propiedad: el informacionalismo. La interrelación entre éste y la economía del conocimiento definen la especificidad de la etapa actual y en esa medida constituye el eje de las nuevas contradicciones creadas por la aparición de fuerzas productivas superiores.

Palabras clave: cambio histórico, capitalismo mundial, cambio tecnológico, economía del conocimiento, infomacionalismo.

\footnotetext{
* Este trabajo se desarrolló como parte de los proyectos de investigación El aprendizaje tecnológico en México dentro del nuevo patrón tecno-industrial mundial y del nuevo ciclo industrial (perteneciente a PAPIT/UNAM) y La nueva economía del conocimiento y el aprendizaje en México. Un estudio comparativo y sectorial (perteneciente a CONACYT).

** Profesor de la Facultad de Economía, UnAM. Correo electrónico: mriver@servidor.unam.mx
} 


\section{Abstract}

The article involves a discussion on the historical change of capitalism and the role played in that process by the emergence of the so-called knowledge economy. It lays down a theoretical and historical framework and proposes a number of hipotheses which together enable us to characterize the specificity of the present stage of capitalism. The knowledge economy is established on the epistemological transformations in technology and in a dramatic reduction in the social costs of accessing useful knowledge. The technological rents that emanate from the diffusion of the new paradigm and the constitution of its industrial pattern necessitate the appearance of a new regime that makes it possible to reorganize informatics property relations. The interrelations between this and the knowledge economy define the specificity of the present stage and it is that measure which constitutes the axis of the new contradictions created by the appearance of superior productive forces.

Keywords: historical change, world capitalism, technological change, knowledge economy, informatics.

\section{Résumé}

Cet article traite de la discussion sur le changement historique du capitalisme et sur le rôle que joue dans ce processus l'émergence de ce qu'on appelle l'économie des savoirs. On formule un cadre théorique et historique et on propose plusieurs hypothèses dont l'intégration conduit à la caractérisation de la spécificité de l'étape actuelle du capitalisme. L'économie des savoirs s'instaure sur des transformations épistémologiques dans la technologie et sur une réduction dramatique des coûts sociaux d'accès aux savoirs utiles. Les revenus technologiques qui émanent de la diffusion du nouveau paradigme et de la constitution de leur patron industriel font apparaître comme nécessaire l'apparition d'un régime nouveau qui permettra un réaménagement des relations de propriété: l'informationalisme. L'interrelation entre ce dernier et l'économie des savoirs définit la spécificité de l'étape actuelle et c'est cette mesure qui constitue l'axe des nouvelles contradictions crées par l'apparition de forces productives supérieures.

Mots clés : changement historique, capitalisme mondial, changement technologique, économie des savoirs, informationalisme.

\section{Resumo}

O artigo fala da discussão sobre a mudança histórica do capitalismo e o papel que tem nesse processo a emergencia da chamada economia do conhecimento. Formula-se um marco teórico e histórico y se poropõem várias hipóteses cuja integração conduz a caraterizar a especificidade da etapa atual do capitalismo. A economia do conhecimento instaura-se sobre transformações epistemológicas, na tecnología e numa redução dramática dos costos sociais do acesso ao conhecimento útil. As rendas tecnológicas que vêm da difussão do novo paradigma e a constituição do seu padrão industrial, faz necessária a aparição dum novo regime que possibilite reordenar as relações de propriedade: o informacionalismo, a inter-relação entre este e a economia do conhecimento definem a especificidade da etapa atual e essa medida constitui o eixo das novas contradições criadas pela aparição de forças produtivas superiores.

Palavras chave: mudança histórica, capitalismo mundial, mudança tecnológica, economia do conhecimento, informacionalismo.

\section{Dêsarrollo}




\section{Ubicación}

a aparición de una tecnología revolucionaria y su constitución posterior como sistema genérico está provocando un cambio en el curso histórico tal y como viene aconteciendo desde principios del siglo xx. Profundizando ese cambio de curso, en los años noventa tendió a consolidarse un nuevo patrón industrial en el sentido de integración de nuevas industrias, familias de productos y estructuras de mercado y consumo, en tanto que, en correspondencia con lo anterior, las viejas industrias han comenzado a rejuvenecerse gracias a la aplicación de la tecnología digital y están integrándose al nuevo modelo industrial. De forma paralela, se difunde mundialmente un tipo de organización productiva que ha convertido en obsoletos los principios y métodos fordistas; además, corresponde con una renovación del perfil obrero en el cual se integran nuevos conocimientos y prácticas laborales que desplazan a las que rigieron en el periodo concluido en los setenta.

En otros campos de la actividad mundial, la transformación ha sido menos concluyente e, incluso, disruptiva y socialmente muy onerosa. Lo anterior sugiere que la revolución tecnológica impone a las restantes estructuras de la reproducción social una secuencia de cambio desigual, con patrones de fuerte polarización y dentro de un marco de coexistencia de elementos en gestación con viejas estructuras semirrenovadas. La confrontación entre lo nuevo y lo viejo llega a ser tan intensa, que se verifican procesos de destrucción creativa, pero también pueden subsistir los desacoples y el ritmo de cambio volverse más lento o ser susceptible de brotes violentos o discontinuos. Cualquiera que sea el ritmo, el proceso innovador provoca incompatibilidades, degrada conocimientos y habilidades, desestabiliza la organización de la producción y crea nuevos problemas de coordinación. ${ }^{1}$

Abarcar este proceso y extraer una propuesta interpretativa acerca de la naturaleza de la etapa actual plantea diversos problemas metodológicos centrados en determinar si la discontinuidad histórica y los rasgos emergentes aparecidos, sobre todo en la década pasada, dan una nueva especificidad a la reproducción social en su conjunto. El concepto de

\footnotetext{
Ésta es una primera aproximación a la naturaleza del cambio histórico, el cual implica procesos prolongados y complejos, que parten de un núcleo inicial de propagación. Éste se encuentra en la constitución y difusión de un nuevo paradigma tecnológico. Este núcleo se va constituyendo sobre la marcha, buscando la complementariedad con las restantes estructuras de la reproducción social a las que va destruyendo o readaptando a sus necesidades. La destrucción implica los sistemas tecnológicos preexistentes, que se vuelven obsoletos. Ciertas instituciones y capacidades, frecuente mente centralizadas en el Estado, pueden limitar el impacto de la destrucción creativa, pero también pueden bloquear temporal o parcialmente el proceso de innovación.
}

\section{Desaarrollo}


especificidad histórica, complementado con el de cambio histórico, constituye el eje para abordar la discusión de la etapa actual, pero debe ir precedida por una cuidadosa aproximación que relacione teoría e historia en el pensamiento social. Habría que empezar por reconocer que el estudio de la especificidad y el cambio históricos es tema controversial signado por innumerables lagunas y sobremarginado de la ortodoxia del pensamiento económico. Sin embargo, esa situación ha cambiado ostensiblemente desde los noventa, conforme a las exigencias analíticas de los nuevos procesos y tendencias; del mismo modo, la necesidad de reemplazar estrategias que han quedado obsoletas provoca la creciente ruptura de los modelos ortodoxos.

En este replanteamiento, la obra de Hodgson, en particular, How Economics Forgot History, tiene el mérito de integrar las aportaciones de Marx, la escuela histórica alemana y los institucionalistas norteamericanos, para cuestionar el modelo ahistórico neoclásico y efectuar una propuesta centrada en el concepto de especificidad histórica.

En la primera parte de este artículo se retoma esa propuesta y se intenta integrarla a la discusión; en la segunda parte, se abordan dos corrientes que Hodgson no consideró: el regulacionismo francés y la teoría de las ondas largas y las revoluciones tecnológicas de la escuela de Sussex. Ambas representan los esfuerzos más amplios efectuados en el último cuarto del siglo xx para concebir sistémicamente el desarrollo del capitalismo y captar su dimensión histórico-estructural. Como veremos, al ampliar el espectro de la discusión se convalida la idea, sugerida por Hodgson, de que hay una línea fundamental de continuidad entre Marx-Veblen y los autores que escriben a fines del siglo xx. ${ }^{2}$

El objetivo del presente artículo es, en consecuencia, abordar la discusión acerca de la especificidad histórica del capitalismo y efectuar una propuesta interpretativa concerniente a la etapa actual, relacionando dos categorías claves que se han propuesto para su estudio: el informacionalismo y la economía del conocimiento (aún no conciliadas teórica e históricamente). Para alcanzar este objetivo se requieren tres mediaciones: a) mostrar la necesidad de las leyes específicas en el estudio de los fenómenos sociales; $b$ ) ubicar las dos principales aportaciones al estudio del cambio histórico del capitalismo efectuadas a fines del siglo xx (el regulacionismo francés y la teoría de las ondas largas y las revoluciones tecnológicas de la escuela de Sussex), para tratar de integrar sus aportaciones desde la perspectiva del materialismo histórico de Marx, y $c$ ) como preludio a la propuesta central, es decir, la caracterización de la etapa actual, se hace un breve examen histórico de la sucesión de las eras tecnológicas para encontrar los factores de continuidad y preparar así el terreno para la conceptualización que se ofrece en el último apartado.

2 Ello no equivale a desconocer la extraordinaria aportación de Gramsci al estudio del cambio histórico; parte de su propuesta tiene gran afinidad de orientación con el institucionalismo norteamericano, como lo sugiere su obra Americanismo y Fordismo (1972, en particular pp. 285 y 287). Gramsci integra el cambio de lo que hoy llamamos base técnico organizativa (el fordismo) con la estructura de institucional (americanismo) para plantear el requerimiento más general del cambio histórico. 
Queda claro, por razones obvias, que para alcanzar los objetivos del presente trabajo se requiere la revisión e incorporación de una muy amplia bibliografía, pues la que se ofrece a lo largo del texto es sólo una parte de la considerada básica para fundamentar las principales hipótesis; sin embargo, el espacio para mejorar el tratamiento bibliográfico es todavía considerable.

\section{La historicidad en las ciencias sociales: una panorámica}

Con la aceleración del progreso material a partir del último cuarto del siglo XIX, la ciencia, como estructura sistemática de conocimiento, empezó a adquirir un papel central en la producción social en detrimento del conocimiento empírico que dominó los primeros avances que dieron origen al capitalismo industrial a fines del siglo xvIII (Mowery y Rosenberg, 1993:6-7). Una consecuencia fue que a partir de mediados del siglo xIX, el conjunto de las disciplinas científicas aceleraron su constitución en torno a ejes epistemológicos que respondieran a los principios de rigor científico que demandaba la aceleración del progreso material. En este proceso, el caso de la ciencia económica destaca entre el resto de las ciencias sociales.

Para representar formalmente el comportamiento del sistema económico la economía tuvo dos opciones; una fue adoptar el enfoque mecánico derivado de la física newtoniana y la otra adoptar el enfoque organicista o evolutivo derivado de la biología darwiniana (Clark y Juma, 1988:199; Hodgson, 1999:87-88; Morowski, 1989:375). En el primero, el todo constituye una estructura estable orientada al equilibrio, cuyas partes y componentes no tienen influencia recíproca ni están determinados por la totalidad (Hodgson, 1999:95). En el enfoque organicista, en cambio, hay una mutua interacción entre el todo y las partes que le confiere a la totalidad una propensión al desequilibrio.

Los economistas clásicos tendieron a oscilar entre ambos enfoques. Malthus fue un precursor de las ideas orgánico-evolutivas, como lo revela su influencia intelectual en Darwin. Pero Smith y otros clásicos se sintieron fuertemente atraídos por la noción de orden en el sistema económico, que era para ellos un reflejo del orden en el mundo físico (Hodgson, 2001:5). La metáfora de un orden económico como proceso gravitacional también se encuentra en Ricardo (ibid.). Como se sabe, a partir de Marshall, esas diferencias de concepción se ahondan para dar lugar a enfoques disciplinarios contrapuestos: a) uno surgido de la escuela neoclásica, queda representado en el marginalismo, la teoría subjetiva del valor y el modelo de equilibrio general; $b$ ) otro que tiende a integrarse en torno al institucionalismo norteamericano, con el liderazgo intelectual de Veblen, quien llamó a refundar la ciencia económica sobre bases evolutivas (Veblen, 1998/ 1919:410-411).

El modelo disciplinario derivado de la física se impuso finalmente en los años veinte, luego de que el institucionalismo norteamericano se desplomó bajo el peso del rechazo

\section{Desaarrollo}


social e intelectual contra el darwinismo social, con el que incorrectamente se le relacionó (Hodgson, 1999:88). El pensamiento neoclásico mantuvo el status de paradigma dominante hasta los años setenta, cuando empezó a sufrir una serie de fracturas internas que tendieron a invalidar su armazón epistemológica. ${ }^{3}$ Ese largo reinado tuvo consecuencias determinantes en la formulación de los principios económicos, entre los cuales sobresale la relación entre leyes o teorías generales en contraposición con las leyes o teorías particulares, de las que depende la especificidad histórica de los fenómenos socioeconómicos.

\section{Teoría general y especificidad histórica}

El pensamiento neoclásico articuló su metodología de la siguiente manera. Partiendo de su marco mecanicista, se representaba al sistema económico como consistente en unidades autónomas de producción y unidades de consumo, que intercambian para equilibrar perfectamente los mercados, haciendo desaparecer los problemas históricos (ya que el tiempo queda abolido). Dicha representación sería incuestionable si fuera modelo ideal de cómo funciona el sistema económico dentro del marco de una serie de supuestos altamente restrictivos, resultado de la abstracción de elementos del proceso real. Pero dicho modelo ideal se convirtió en marco referencial en el sentido de que servía para determinar la viabilidad de los procesos reales (Hodgson, 2001:16). En otras palabras, un conjunto de abstracciones lógicas se convierten en modelo normativo para determinar no el ser, sino el deber ser.

Como explica Hodgson, en la conversión del modelo lógico en marco normativo, el principio de ley o teoría general ocupa un lugar central. Al adoptar una cosmovisión, cualquiera que ésta sea, una disciplina científica tiene que apoyarse en leyes generales en el sentido de estructuras explicativas que tengan nivel absoluto de aplicabilidad y, en esa medida, unifiquen la comprensión de los fenómenos que abarca. Está por demás insistir en la importancia de las leyes generales para sustentar el pensamiento científico, pero es crucial la manera cómo se llega a ellas. La formulación de una ley general sigue dos caminos (Hodgson, 2001:10). De una parte hay un camino lógico o derivacional que significa que cada vez más conclusiones de una disciplina derivan de un mismo conjunto de axiomas; como se advierte, esta unificación explicativa implica simples conexiones deductivas entre los axiomas y las conclusiones. De otro lado tenemos la unificación analítica, la cual exige una identificación de los rasgos comunes de los fenómenos como limitación a cualquier conclusión general sobre su naturaleza (ibid.). La diferencia entre ambos tipos de generalización teórica es que, en el primer caso, estamos frente a un ejercicio lógico-deductivo, y en el segundo, ante un proceso exploratorio, en la cual la prio-

3 Éste es el momento en que la teoría de los juegos entra a desplazar al modelo walrasiano de equilibrio general como base de la racionalidad neoclásica (Hodgson, 2001:18). 
ridad la tienen las características reales de las entidades y no las proposiciones (Hodgson, 2001:11).

La teoría del equilibrio general, como la estructura fundamental sobre la que se sostiene el pensamiento neoclásico, corresponde al primer tipo de generalización científica. Las consecuencias metodológicas son muy variadas, pero una de las más importantes, por lo menos para los fines asumidos en este trabajo, es la abolición del tiempo histórico. Es posible llegar a esa conclusión después de examinar varios atributos del modelo: una es restar importancia al proceso real y centrarse en un conjunto de rasgos abstractos que permanecen inalterables, lo que da lugar a una replicabilidad continua de sus atributos; finalmente, no hay espacio para leyes específicas, ya que el comportamiento de los fenómenos está totalmente unificado; en consecuencia, no hay historia.

Las leyes o teorías específicas constituyen otro nivel en el cual se representan las regularidades del mundo real, pero subordinado a la evolución de los fenómenos y, por ende, a la aparición de propiedades emergentes que modifican su naturaleza (Hodgson, op. cit.: 12-34). Si el modelo ontológico de la física abolió el tiempo histórico y con él las leyes específicas de movimiento, el método inspirado en la biología propone su restitución al ver el proceso real como evolutivo. A continuación veremos cómo antes de la llamada revolución marginalista la economía política percibió la especificidad histórica de los fenómenos sociales y formuló generalizaciones para explicar el movimiento real.

Como señala Hodgson (op. cit.:44), a principios del siglo xIX se sentaron las bases para ligar el término tiempo histórico con el de progreso social, dejando atrás las concepciones circulares de la evolución humana. La noción básica de progresión de la condición humana (o espíritu) provino de Hegel, a partir de quien derivaron dos propuestas para el estudio del cambio histórico (ibid.): una de ellas se encuentra en Marx y la otra en la escuela histórica alemana (ЕHA). Aunque ambas tienen un punto de partida común, siguen líneas metodológicas divergentes, ya que la EHA, en su rechazo al deductivismo de la economía clásica, se inclina hacia el empirismo y el idealismo (Hodgson, ibid:58), en tanto que Marx establece las bases materialistas para el estudio del cambio histórico social, combinando la formulación de leyes que operan en tres distintos niveles.

Desde el punto de vista del estudio de los procesos históricos, la aportación fundamental de Marx que, a su vez, constituye la base de su concepción materialista de la historia es que el progreso material de la humanidad implica el pasaje de un modo de producción a otro; ${ }^{4}$ ese paso histórico resulta de contradicciones internas en el nivel de la estructura

\footnotetext{
4 Como en otros conceptos o estructuras teóricas, el materialismo histórico de Marx (y de Engels) no está expuesto sistemáticamente. La principal exposición, aunque esquemática, es el prólogo de la Contribución a la crítica de la economía política, que se cita aquí de manera preferente, específica mente la página 343. Obviamente el Manifiesto comunista, con sus limitaciones, es también una fuente valiosa. En la Ideología alemana está solidamente argumentada la concepción materialista del desarrollo social (véanse, en particular, pp. 39 42, 55 y ss); en el prefacio a la primera edición de
}

\section{Desaarrollo}


fundamental, que se resuelven en una nueva síntesis, implicando un salto cualitativo en las relaciones sociales, núcleo de aquella estructura fundamental (Marx, s/f., p. 343). Los tres niveles teóricos que se combinan en esta propuesta son los siguientes: ${ }^{5} a$ ) uno corresponde a principios transhistóricos (la sobrevivencia colectiva implica un control creciente de la naturaleza); b) otro corresponde a leyes o categorías generales (en determinado nivel de desarrollo las fuerzas productivas sociales entrarán en contradicción con las relaciones específicas de producción en cuyo seno se gestaron); c) finalmente están las leyes específicas, válidas exclusivamente para un modo de producción históricamente definido, como las teorías del valor, de la acumulación (entre otras) propios del capitalismo (Marx, 1946, t. I, p. XIII).

También habría que subrayar que el padre del materialismo histórico legó un aparato analítico-conceptual para el estudio de la transformación del capitalismo, estructurado en distintos niveles en su obra fundamental, El capital. De una parte, tenemos el estudio del pasaje de la plusvalía absoluta a la relativa o, lo que es lo mismo, específicamente, la sustentación del régimen capitalista de producción (t. I, sección quinta). En su análisis de la ley de la acumulación capitalista (t. I, cap. xxIII, caps. 1 a 3) Marx la presenta como el proceso que encadena las transformaciones del modo de producción capitalista (creciente centralización del capital como expresión del pasaje en niveles de mayor madurez estructural y, consustancial a lo anterior, el aumento de la composición orgánica).

Sin embargo, la aportación de Marx, que constituyen la más valiosa herramienta para el estudio del cambio histórico-social, tiene tres limitaciones:

a) el sesgo determinista y teleológico de parte de su obra, la cual implica una negación de los principios de indeterminación que deben fundamentar la visión del proceso histórico;

b) la insuficiencia del nivel de teorización específica para abarcar las transformaciones históricas del sistema capitalista, que ha mostrado una extraordinaria capacidad de adaptación a lo largo del tiempo;

c) la relativa indeterminación de la relación entre agente y estructura, derivada de la desconexión de la superestructura, lo cual deja ciertos vacíos en la explicación de la causalidad social.

Veblen intentó combinar críticamente las aportaciones de Marx y las de la EHA para generar una alternativa a la economía no evolucionista y ahistórica en proceso de consoli-

El capital, Marx formula su programa teórico para estudiar el capitalismo a partir de sus categorías específicas, leyes del valor, de la plusvalía, entre otras (p. XIII). Por limitaciones de espacio no se citó el Antidühring de Engels, que no debiera estar ausente en un tratamiento más detallado que el actual.

5 Adaptando la propuesta de Hodgson (ibid.:45 46).

\section{Dêsaarróllo}


dación disciplinaria desde 1870 (Veblen, 1998/1919; Hodgson, ibid.:140). Su llamado daba eco al programa de la EHA centrado en los siguientes puntos:

a) descartar el uso exclusivo de métodos deductivos y reconocer la importancia del tratamiento histórico y la convalidación estadística;

b) negar la existencia de leyes inmutables en la economía, subrayando la historicidad que implica aproximaciones teóricas específicas a etapas o condiciones espaciales diferenciadas;

c) rechazo a la explicación del comportamiento económico a partir de modelos de racionalidad apriorística. ${ }^{6}$

En lo que concierne al estudio de la especificidad histórica del capitalismo, Veblen razonó en el marco del materialismo histórico de Marx, pero rechazó su aplicación determinista y teleológica, a favor de los principios de indeterminación y de causación acumulativa (1919/2003:416 y 436). Igualmente, desde el punto de vista del estudio de la estructura o sistema económico, reformuló la relación entre sujeto y estructura, asumiendo que las acciones individual y social están determinadas fundamentalmente por hábitos de pensamiento y acción (Veblen, 1963/1899:194-198). Tales hábitos constituyen la base del término instituciones que juegan el papel de conductos de la acción social y, por ende, del movimiento histórico. Este replanteamiento sentó las bases de una revolución en la concepción de cambio histórico, ya que propuso una idea básica de su concreción, interrelacionando condiciones materiales e instituciones, en el sentido de la adecuación y posterior inadecuación entre ambas, con el peso de la inercia institucional (Veblen, 1899/ 1963:194).

Nótese que Veblen, inspirándose en Marx, asume que las relaciones sociales tienen varios componentes que ejercen una función distinta en la reproducción social. Aunque no hizo explícita su concepción integral de las relaciones sociales, sugirió que las instituciones son un elemento primordial y foco de la contradicción que genera el movimiento histórico-social. ${ }^{7}$ La contradicción fundamental se daría, entonces, entre condiciones materiales y hábitos mentales cosificados o instituciones. Lamentablemente, el colapso del proyecto institucionalista de Veblen retrasó la integración de su propuesta, de suerte que una noción más acabada del cambio histórico tuvo que esperar hasta los años setentaochenta (Rivera, inédito).

6 Proviene de Hodgson (ibid.:139).

7 "La estructura social sólo cambia, se desarrolla y se adapta a una situación modificada, mediante un cambio en los hábitos mentales de las diversas clases de la comunidad [...] La evolución de la sociedad es sustancialmente un proceso de adaptación mental de los individuos, bajo la presión de las circunstancias que no toleran por más tiempo hábitos mentales formados en el pasado" (Veblen, 1899/1963:197 198).

\section{Desaarrollo}


Pasaremos ahora a revisar las dos contribuciones más importantes a la teoría del cambio histórico del capitalismo efectuadas a fines del siglo xx. Al confrontar sus aportaciones con los elementos destacados en el apartado anterior, estaremos en condiciones de extraer una propuesta propia para el estudio de la especificidad histórica actual.

\section{El resurgimiento del concepto especificidad y la discusión de cambio histórico}

\section{El regulacionismo francés y las etapas del desarrollo capitalista}

En los años setenta se reabrió la discusión acerca de la transformación histórica del capitalismo desde dos grandes orientaciones que, en lugar de complementarse, tendieron a contraponerse. El estallido de la crisis mundial de principios de los setenta jugó el papel de catalizador del debate. De un lado, se perfiló una interpretación en la cual la crisis mundial ponía de manifiesto las contradicciones terminales del capitalismo y la cercanía de su colapso definitivo. Por otro lado, cobró fuerza, mediante las aportaciones que examinaremos a continuación, la interpretación de que la crisis era, en sí, el vehículo de una transformación histórica del capitalismo, la cual conduciría a una estructuración de sus relaciones internas.

La primera interpretación constituye una aplicación de la hipótesis de que las fuerzas contrarrestantes de la tendencia descendente de la tasa de ganancia se ven crecientemente debilitadas en el largo plazo. En consecuencia, esta interpretación intentaba convalidar, para las condiciones históricas de los setenta en adelante, la tesis de un salto dialéctico en las relaciones de producción, que implicaría en primer lugar la ruina del modo de producción capitalista. El método interpretativo adoptado por autores ortodoxos tendía a negar la especificidad histórica, esto es, asumir que las leyes de movimiento permanecen inmutables y se aplican irrestrictamente del periodo o etapa. Pero este desconocimiento de la especificidad histórica expresa la ya identificada limitación de la propuesta originaria de

Marx (ausencia de un nivel complementario o intermedio de teorización acorde con la prolongación histórica del modo de producción capitalista).

El regulacionismo francés constituye la otra corriente interpretativa que emerge en los setenta y asume como tarea las insuficiencias del nivel intermedio de teorización, es decir, lo que hemos llamado especificidad histórica. En esa medida, esta corriente se contrapone a las generalizaciones excesivas que permeaban las ciencias sociales, tanto fuera como dentro del marxismo. Al respecto, Boyer (1988:69) lamentaba que los esfuerzos teóricos y de análisis se hubieran reducido a verificar el inevitable colapso del capitalismo, debido al cumplimiento de su ley fundamental: la tendencia descendente de la tasas de ganancia. Esa criticable orientación metodológica, señalaba el autor, deriva de la insuficiente integración entre la teoría y el análisis empírico y el predominio de métodos puramente 
deductivos o inductivos (Boyer, 1988:69). Consecuentemente con ese programa de investigación, el regulacionismo constituye un intento por elaborar e integrar en una misma estructura analítica un continuo de conceptos, desde los más abstractos, hasta los que captan la especificidad del comportamiento de los agentes económicos (ibid.).

La teorización intermedia efectuada por los regulacionistas se tradujo en tres importantes innovaciones para el estudio del cambio capitalista y la especificidad histórica de las diferentes fases de su desarrollo:

a) la reelaboración del concepto modo de producción para captar la especificidad histórica de la conformación de las fuerzas productivas y de la estructura social a la cual se integran. ${ }^{8}$ Así surgen los términos régimen de acumulación y modo de regulación. El primero sintetiza los factores específicos que permiten el funcionamiento regular del proceso de acumulación, identificando la conformación técnica y social de su operación. Por su parte, la voz modo de regulación identifica los canales por medio de los cuales se encauza la reproducción económica.

b) La definición del modo de regulación a partir de la influencia del institucionalismo norteamericano, en particular de Veblen. ${ }^{9}$ Esto significa que se asume la idea de que la interacción social está estructurada de manera específica, lo cual implica la existencia de reglas y convenciones asimiladas a las mismas relaciones sociales. Entre los canales que posibilitan la interacción social, los regulacionistas destacan las relaciones crediticias, los nexos capital-trabajo (o relación salarial), el tipo de competencia, la adhesión al régimen internacional y las formas de intervención estatal. ${ }^{10}$

8 Los regulacionistas proponen un doble criterio de periodización: uno, a partir de la sucesión de modos de regulación (de la competitiva a la monopólica); el otro, a partir de la transformación del régimen de acumulación (de la extensiva a la intensiva); véase Boyer (1984:42 y ss). La especificidad histórica de cada fase estaría dada por la interrelación entre ambas estructuras; por ejemplo, en la fase que se abre a partir de 1945 y entra en crisis en los setenta, el modo de regulación está determinado por las instituciones y políticas keynesianas, en confluencia con una acumulación intensiva y consumo de masas. Bajo la inspiración de esta propuesta se generalizó la designación de la fase anterior como "fordista" o capitalismo fordista, véanse Harvey (1990:173 188); Marglin (1990:12 y 23) y Dabat (1993:163 171).

9 Durante los setenta y ochenta los regulacionistas no reconocieron su deuda intelectual con el insti tucionalismo norteamericano; pero en un trabajo de 2001, Boyer se declara implícitamente institucionalista en un sentido vebliano, al señalar: "Desde una perspectiva metodológica [la teoría de la regulación] pertenece [...] a un marco donde los actores se comportan de acuerdo a los que ellos creen son sus mejores intereses, pero dentro de instituciones que han sido construidas por la acción colectiva del pasado" (pp. 115 116). Como se advierte, esa declaración está extraída de la obra central de Veblen de 1899, ya citada aquí.

10 El modo de regulación como sistema institucional constituye un conjunto de reglas que definen el comportamiento individual y colectivo en tres sentidos: a) automatizan el funcionamiento del siste ma socioeconómico en su conjunto; $b$ ) permiten el funcionamiento regular de la acumulación, y c) reproducen las relaciones sociales de manera históricamente determinada (Boyer, 1988, infra p. 75).

\section{Desaarrollo}


c) Ampliación de los alcances del concepto crisis para incorporar el de crisis estructural, que designa los episodios críticos durante los cuales se rompe la conjugación entre modo de regulación y régimen de acumulación, lo cual implica que el sistema socioeconómico pierde su capacidad para reproducirse en el largo plazo. La crisis estructural es, consecuentemente, el mediador del cambio histórico.

Estas tres aportaciones se interrelacionaron para darle nuevo significado a la periodización del desarrollo capitalista, fundada en la configuración e interrelación entre modo de regulación y régimen de acumulación. Los procesos de recomposición económicos y sociopolíticos que se abren a partir de la crisis estructural tendrían que estar enmarcados dentro de la indeterminación para que cada etapa o fase del capitalismo posea su especificidad histórica.

Aunque no estuvo exento de críticas, ${ }^{11}$ la propuesta de concebir el desarrollo dentro del marco de un proceso histórico sujeto a discontinuidades y cortes de tendencias tuvo enorme influencia en el pensamiento social, incluso más allá de las disciplinas heterodoxas. En particular, el estudio del régimen de acumulación intensivo y regulación keynesiana abrió una vertiente de investigación en la que se interrelacionaron diversos planos de análisis (empresarial y organización industrial, gestión pública, tecnología, relaciones laborales, organización del espacio). En las filas de la ortodoxia del marxismo se argumentó que el regulacionismo y la escuela de Sussex estaban presentando al capitalismo como dotado de capacidad endógena para reconstituirse y superar sus contradicciones terminales.

\section{La teoría de las ondas largas y su visión del cambio histórico}

La única propuesta teórico-metodológica que compite con el regulacionismo en cuanto al análisis del cambio histórico es la vieja teoría de las ondas largas, derivada de la obra principal de Kondratiev, publicada en ruso en 1925 y 1926 (Sandoval, 1992:11). La hipótesis indica que la acumulación de capital en la escala mundial sigue un patrón de grandes ondas cíclicas de entre 50 a 60 años de duración, primero de ascenso y luego de descenso, que equivalen a etapas recurrentes del desarrollo capitalista.

Esta teoría tiene su propio historial de controversia en las filas del marxismo desde los años veinte, ya que pone en tela de juicio lo que es, probablemente, la interpretación dominante de la ley de la tendencia descendente de la tasas de ganancia. ${ }^{12}$ Si una onda

11 La más destacada entre ellas es probablemente la de Brenner y Glick (1991), en la cual les reprochan no tomar en cuenta el amplio sistema de relaciones sociales y de propiedad que constituyen el fundamento institucional del paso de una fase del capitalismo a otra (p. 105).

12 Como explica Duijn, las violentas críticas del estalinismo contra Kondratiev tienen que ver con su predicción de una recuperación de larga duración, que seguiría a la onda larga descendente de 1914 1920; los líderes soviéticos habían apostado políticamente en contra de esta posibilidad, lo cual significaba que el punto de vista de Kondratiev chocaba contra el marxismo oficial (1996/1983:79 80).

\section{Dessarras perllo}


larga descendente tiene solución endógena, el capitalismo tendría capacidad para superar sus contradicciones terminales.

Las nociones legadas por Kondratiev sólo constituyen el fundamento general de la teoría actual, ya que ésta experimentó, a lo largo del siglo xx, una transformación a partir de sucesivas aportaciones que definieron con más rigor su marco conceptual y su campo de aplicabilidad. Dicha reelaboración ha dotado a esta teoría de un rango epistemológico diferente al que presenta el regulacionismo, porque ha tendido a convertirse en un modelo teórico para el estudio del crecimiento en condiciones de discontinuidad, por lo cual puede considerarse un tipo sui generis de teoría general, diferente a la propuesta por los franceses. ${ }^{13}$ Por eso la teoría de las ondas largas asume un funcionamiento uniforme de los mecanismos motores del ciclo, en tanto que los regulacionistas, apegados a la tradición neoinstitucionalista, tienden a hacer hincapié en la especificidad histórica de las fuerzas que inducen tanto al auge, como a la declinación y estancamiento de la acumulación de capital. Pese a sus diferencias de método de estudio, ambas propuestas son altamente complementarias, por lo cual era lógico que en cierto nivel de elaboración tendieran a concluir. Ésa es la aportación efectuada en la escuela de Sussex por Freeman y Pérez, la cual dio lugar a un gran avance, pero dejó algunos problemas sin resolver.

Las siguientes son las hipótesis generales que esta teoría fue adoptando para llegar a su conformación final como concepción del crecimiento discontinuo. Primero tenemos la formulación efectuada por Kondratiev en los años veinte; enseguida podemos distinguir una segunda gran formulación efectuada por Schumpeter y, finalmente, una elaboración más reciente originada en la escuela de Sussex, en la que tratan de integrarse aportaciones previas, adoptando como núcleo la concepción del cambio histórico que los franceses propusieron.

Aunque Kondratiev abrió una línea de análisis que resulta controversial para amplios estamentos de la ortodoxia marxista, construye su propuesta a partir de los grandes bloques de la teoría de Marx. ${ }^{14}$ Por una parte, formula la idea de que las ondas expansivas de la acumulación de capital obedecen a los grandes avances en las fuerzas productivas (1925/ 1996:64). Las grandes invenciones científico-técnicas son el fundamento primario de los avances en las fuerzas productivas, pero se requieren condiciones económicas favorables para que esos factores potenciales repercutan en las actividades productivas $y$, de ese modo, se expanda a largo plazo la acumulación de capital (ibid.). En el marco de esas condiciones favorables, la aceleración de la acumulación de capital tiende a ordenar violentamente las condiciones generales de la sociedad, originando grandes conflictos como

3 Véase el esbozo de este deslinde en Boyer (1988:82).

14 Naturalmente, con la excepción del mecanismo explicativo de la crisis, ya que Kondratiev invierte la explicación de Marx al poner la insuficiencia al centro de la inflexión del ciclo y no el exceso de capital. Es decir, añadió un elemento proveniente de Tugan Baranovsky (véase Glasner, 1997:405).

\section{Desaarrollo}


guerras y revoluciones, que expresan indirectamente la lucha por mercados y materias primas (op. cit.:64-65). Si los rasgos dominantes de las ondas largas se definen a partir de la configuración de los avances científico-técnicos, su proceso generador deriva de la lógica de agotamiento y reemplazo de los bienes de capital, es decir, una readaptación de la teoría de Marx sobre ciclos decenales de inversión (Kondartiev, 1926/1992:58-59). Como se advierte, se trata de una interpretación enteramente endógena del mecanismo causal de las ondas largas que abarca el paso de la recesión a la expansión y viceversa.

Aunque en el tiempo de Kondratiev no se había profundizado en la diferencia entre invención e innovación y entre conocimiento científico y tecnológico, su análisis apunta a que los avances científico-técnicos pueden permanecer en estado latente por un tiempo considerable, a menos que haya condiciones favorables para su aplicación, en otras palabras, que existan prospectos favorables para elevar la rentabilidad capitalista que dependan de una configuración socioinstitucional determinada. En resumen, Kondratiev, siguiendo a Marx, intenta presentar un análisis integrado entre factores técnicos (fuerzas productivas) y sociales (relaciones de producción vistas como condiciones sociales).

En manos de Schumpeter (1939/1964:143-150) la teoría de las ondas largas experimenta un extraordinario aunque desigual avance. Su principal mérito radica en haber situado su análisis en lo que hoy llamaríamos un marco institucionalista; es decir, cómo el funcionamiento interno del capitalismo se modifica según ciertos rasgos o predisposiciones emanadas de su propia estructura. Aquí, en cierto modo se aproxima a Marx, al presentar al empresario como impulsado por inclinaciones subjetivas que son el resultado de la estructura económica. ${ }^{15} \mathrm{Su}$ otra gran aportación está en el terreno de la teoría de la innovación para explicar los ciclos de crecimiento del capitalismo, en el cual la discontinuidad es una de las principales características; en ese sentido, desarrolla la idea original de Kondratiev. ${ }^{16}$ Las innovaciones generan ondas expansivas porque después de que han sido introducidas, debe haber una pausa para traducirse en nuevos productos o reducir el costo de los existentes (Schumpeter, 1964:141). Dicho de otro modo, cuando las innovaciones llegan al mercado, se requiere un proceso de adaptación o de recesión que constituye el inverso de la onda expansiva causada por la propagación inicial de las innovaciones (ibid.).

$\mathrm{Al}$ sugerir que las innovaciones se prestan discontinuamente, Schumpeter dio un salto en la teoría de las ondas largas pero, al mismo tiempo, abrió numerosos interrogantes de los porqués se agrupan. De cualquier modo, el trabajo de Schumpeter dejó planteada la

15 Así quedan sentadas las bases para analizar la innovación empresarial, pero el empresario se desempeña dentro de una estructura social que condiciona su conducta y que puede evadirse o romperse mediante la misma conducta social.

16 Con la diferencia de que en lugar de los ciclos de reinversión de Kondratiev, Schumpeter introduce la teoría de la innovación que contempla los procesos de difusión: "los nuevos métodos son copiados y mejorados; la adaptación a ellos o a los impactos de las nuevas mercancías consiste en crear innovaciones inducidas" (op. cit::119). 
base para relacionar la idea de que las innovaciones aparecen discontinuamente y en racimo con el concepto ciclo de vida, dentro del marco de una nueva teoría del crecimiento discontinuo del capitalismo.

Aproximación entre la teoría de las ondas largas

y el enfoque regulacionista

Trabajando con este marco teórico, los seguidores de Schumpeter trataron de encontrar una solución a los problemas de integridad teórica, causados por el planteamiento de que las innovaciones tecnológicas son el motor de las ondas largas. El problema central es la explicación de por qué las innovaciones tienden a agruparse y constituir la base de la dinámica del capitalismo. Mensch (1979:123) afinó la teoría de la discontinuidad de las innovaciones (distinción entre menores y centrales) y trató de probar empíricamente que las innovaciones mayores se agrupan en los periodos de depresión para impulsar el crecimiento económico. Su trabajo fue criticado ampliamente, pero fue la base para una nueva propuesta acerca del mecanismo causal del ciclo ondulatorio a largo plazo.

Primero Freeman (1984:497), después Pérez (1983:361-366) y luego Freeman y Pérez (1988:46-49) argumentaron que las innovaciones mayores o radicales pueden presentarse de manera dispersa en el tiempo y tienden a integrarse conformando sistemas o constelaciones y después comienza su difusión como revolución tecnológica (Freeman y Pérez, 1988:49). El proceso de difusión da lugar a patrones de crecimiento del tipo presentado por Kondratiev (Pérez, 1983:375). Este paso en el análisis es producto de una interesante convergencia entre la teoría de las ondas largas en la línea abierta a partir de Schumpeter con las aportaciones del regulacionismo francés, proponiendo el concepto paradigma tecnoeconómico. En este nuevo término subyace la noción de régimen de acumulación, pero coronada por la idea de que lo determinante en la apertura de una onda ascendente es la organización de un conjunto de principios tecnológico-organizativos como práctica óptima o nuevo saber social, el cual servirá para generar una trayectoria de nuevos productos y procesos (Freeman y Pérez, 1988:47). Igualmente, la categoría de sistema socioinstitucional es una reelaboración de la noción francesa modo de regulación, que se suma a la visión de Veblen de que la acción social debe estar estructurada para dar lugar al dinamismo, sin abandonar la idea de contradicción representada en la desigualdad de los ciclos de vida de instituciones y tecnología.

Así integrada, la teoría de las ondas largas puede constituirse en una poderosa herramienta para el estudio del cambio histórico del capitalismo. No obstante, esta nueva versión de la teoría de las ondas largas deja sin resolver algunas cuestiones medulares. Primero asume acríticamente la hipótesis de Kondratiev de que las ondas largas presentan patrones regulares de entre 50 y 60 años. Segundo, en conexión con lo anterior propone, siguiendo la tradición regulacionista, un modelo teórico de desadecuación-adecuación entre el paradigma

\section{Desaarrollo}


tecnoeconómico y el régimen socioinstitucional que deja poco margen a la indeterminación histórica (Tylecote, 1991:18-35). En cuanto a lo primero, suponiendo que la acumulación de capital avanza a grandes saltos históricos mediados por contracciones de larga duración, es problemático asumir patrones temporales uniformes. En particular, la convalidación empírica del tercer Kondratiev ha sido fuertemente cuestionada ${ }^{17}$ y cabe la posibilidad de proponer una relación teórica diferente entre la onda ascendente del tercer K (que va de 1895 a 1929) y la onda ascendente del cuarto K (de 1948 a 1973). Según esa interpretación alternativa, ${ }^{18}$ las innovaciones que aparecieron desde los años setenta del siglo XIX (primero el acero barato, luego el dinamo eléctrico, el motor de combustión interna y la cadena de montaje) constituyen una constelación que se integra progresivamente para dar lugar a lo que Pérez llama después ondas de crecimiento: hubo una primera onda de crecimiento irregular entre 1895 y 1929 (con dos subondas: 1895-1913 y otra un poco más débil entre 1920 y 1929). ${ }^{19} \mathrm{Al}$ aparecer, diversos obstáculos sociales, institucionales y de equilibrio de poder provocan un colapso mundial que lleva a la gran reestructuración institucional asociada al New Deal y Bretton Woods. En dicha perspectiva, la edad de oro representaría lo que Pérez (2001:123) posteriormente llama fordismo maduro.

Algo semejante parece suceder a partir de fines de los cuarenta: las innovaciones de la era electrónico-informática se entrelazan, a partir de los años setenta del siglo xx, para generar ondas de crecimiento. En los noventa tenemos la fase de crecimiento temprano que quedaría delimitada por la crisis mundial 2001-2003. Una segunda onda de crecimiento (tardía) podría depender de una reestructuración equivalente a las décadas de los treinta y cuarenta, para de allí prolongarse en medio de transformaciones más profundas del capitalismo.

Estos problemas de conjugación entre teoría general, teoría específica y evidencia empírica parecen dejar tres medios de solución: una, mantener la teoría de las ondas largas, ajustándola mediante una propuesta intermedia concerniente a flexibilizar la relación entre el paradigma tecnoeconómico y el sistema socioinstitucional, como propone Tylecote (1991:20-24); la segunda, descartar la teoría de las ondas largas a favor de otra centrada en la difusión de las revoluciones tecnológicas, como efectivamente hace Carlota Pérez (2002:60-61). Finalmente, tratar de integrar los cambios en la tecnología con los correspondientes a la organización sociopolítica y de clase, para dar paso a lo que podemos llamar teoría de las fases..$^{20}$

17 Probablemente Solomou (1988, especialmente cap. 8) sea el más conocido entre estos críticos, quien no sólo subraya la inexistencia del tercer Kondratiev, sino que sugiere que el cuarto, correspondiente al fordismo, es producto de una excepcionalidad histórica.

18 De hecho, esbozada por Pérez ( 2001), pero sobrepuesta a su teoría más reciente de propagación de las revoluciones tecnológicas (2002).

19 Para las tasas de crecimiento de la primera onda, véanse Tylecote (op. cit.:12) y Solomou (op. cit.: 28 y ss).

20 Es la propuesta de Dabat (1993, cap. VII, principalmente pp. 163 167) y ampliada en 2002 (al integrar al análisis la estructura espacial del capitalismo, véase pp. 67 81). En lugar de asimilarse a 


\section{Integración de categorías para el estudio de cambio histórico $y$ especificidad histórica}

$\mathrm{Al}$ integrar las distintas aportaciones efectuadas por las dos corrientes previamente discutidas, podemos establecer las siguientes hipótesis generales para la caracterización del cambio histórico:

a) El desarrollo de las fuerzas productivas en el capitalismo sigue una secuencia continua (innovaciones que no implican la destrucción de los sistemas existentes), pero también otra discontinua, representada por la sucesión de revoluciones tecnológicas, que constituyen el fundamento material para el pasaje de una etapa histórica a otra;

b) Siendo el epicentro de la transformación de la base económica del capitalismo, la revolución tecnológica se propagará al conjunto del sistema productivo y social, mediante una adecuación contradictoria y desigual de las restantes estructuras de la reproducción social, en particular del sistema socioinstitucional;

c) el sistema socioinstitucional es seleccionado por la tecnología, pero tiene la capacidad para reactuar sobre este último, lo cual sitúa el cambio tecnológico en el marco de una causación multilineal;

d) si bien, como consecuencia de la interrelación entre tecnología e instituciones se abren trayectorias de crecimiento a largo plazo, su durabilidad y consolidación es indeterminada, en virtud de que las crisis estructurales que acompañan su evolución tienen escenarios abiertos.

A partir de estas cuatro hipótesis generale ${ }^{21}$ pueden replantearse los fundamentos de la dinámica del capitalismo, lo cual constituye, en conjunto, el marco referencial básico a partir del cual puede determinarse el funcionamiento de sus leyes específicas. En consecuencia, la forma en que se expresen las leyes específicas formuladas por Marx debe definirse mediante la investigación histórico-concreta. Adicionalmente, esa relación entre las tendencias generales de comportamiento y su concreción histórica debe apoyarse en un marco analítico capaz de explicar las relaciones causales que gobiernan el comportamiento de las revoluciones tecnológicas y su relación con el resto de las instancias de la reproducción social.

Para identificar los bloques de este marco analítico habría que adoptar la concepción de revolución tecnológica propuesta por Freeman y Pérez. Ambos señalan que las innova-

la tradición estructuralista francesa de segmentar la realidad en estructuras (tecnológica y socio institucional), propone la idea de transformación o destrucción gradual de la estructura social y espacial, conforme se renueva la base productiva, sin descartar la noción de salto histórico (pasaje del cambio de cantidad al de calidad).

21 Tienen esa condición general porque son aplicables al comportamiento del capitalismo en cualquiera de sus fases históricas.

\section{Desaarrollo}


ciones aparecen de manera dispersa en el tiempo, pero tienden a unirse como sistema en torno a las que tienen efectos radicales. Una vez que comienzan a integrarse, se da su propagación al resto de la estructura productiva y social. Tal unión, como sistema, prefigura un paradigma tecnoeconómico o tecnología genérica, el cual constituye una unión de fuerzas productivas y relaciones de producción, que proporciona un primer nivel de estructuración de fuerzas motoras de largo plazo.

Pero, por qué el desarrollo de las fuerzas productivas adopta la forma predominante de revoluciones tecnológicas. Sólo recientemente se ha asumido esta pregunta como objeto de teorización. El punto de partida es que el capitalismo ha alcanzado, desde la revolución industrial, una estructuración institucional que expresa su propensión al dinamismo ${ }^{22}$ dentro de una lucha perenne entre estabilidad e inestabilidad. Esa dicotomía tiende a expresarse mediante la relación entre inercia y dinamismo. Recordemos que el cambio tecnológico es una composición de diversas formas de conocimiento útil. A su vez, ese conocimiento puede clasificarse en dos grupos: de primer y de segundo orden (March, 1996:102). El primero se refiere al que se utiliza para hacer o producir las cosas conocidas; el segundo, para hacer nuevas cosas. El de primer orden resulta de la codificación y estandarización del conocimiento tecnológico producido por una revolución tecnológica previa; es decir, es la consolidación del conocimiento de segundo orden (ibid.), cuya supremacía del desarrollo histórico social le corresponde.

Podemos explicar el paso de una revolución tecnológica a otra como producto de la interrelación entre ambos tipos de conocimiento; si, a su vez, adoptamos una concepción de ciclo vital, podremos explorar la relación consolidación-inercia y generalizacióninvención siguiendo la propuesta de Nooteboom (2000:178-183). Lo que hoy constituyen procesos probados y rutinarios que brindan un nivel de rendimiento determinado son, primero, el resultado de saltos o revoluciones en el stock del conocimiento útil, la primera transformación que experimentan es su consolidación, por medio de la cual se convierten en paradigmas, diseños dominantes o regímenes tecnológicos (op. cit::172). Pero si la consolidación es necesaria para una explotación eficiente, tiende a resultar inercia que expresa la adhesión rutinaria a las prácticas conocidas, después de que éstas han adquirido existencia propia. ${ }^{23}$ Lo anterior, también obedece a una consideración práctica de supervivencia: como argumenta Nooteboom, puede ser contraproducente desechar una práctica demasiado pronto, antes de conocer sus límites y posibilidades de reemplazo (p. 183). Eso

22 Mokyr (2002:31) señala que, a partir de la revolución industrial, confluyeron factores socio económicos e institucionales que rompieron la retroalimentación negativa que limitaba el crecimiento en el largo plazo. Volveremos a este punto más adelante.

23 Esto coloca la noción de inercia de las organizaciones en una nueva perspectiva, a éstas se les requiere no sólo para coordinación y control, es decir, para lograr una explotación eficiente. Representa, al mismo tiempo, la adhesión al principio de la preservación, pero el conservadurismo puede convertirse en excesivo y bloquear la innovación (Nooteboom, op. cit.:177). 
lo confirma Kuhn al señalar que la ciencia normal no se desecha hasta que es abrumadora la evidencia de que existen anomalías (Kuhn, 1971:225). Ello implica que debemos agotar nuestras teorías no sólo para recuperar nuestra inversión, sino para acumular la necesaria motivación para pasar a una alternativa. Pero al mismo tiempo, el paso a un conocimiento de segundo orden debe ser precedido por una amenaza a la existencia actual o un desplome del rendimiento por debajo de ciertos límites, que se produce como agotamiento del conocimiento de primer orden (Nooteboom, op. cit::176).

La idea básica que deriva de lo anterior es que antes de que podamos reemplazar una práctica — sea una teoría, tecnología u organización-, primero debemos exprimir su potencial; esto es necesario porque es el trampolín para el cambio, para descubrir los límites de la práctica actual y para saber cuáles elementos preservar. A medida que la aplicación de una práctica se extiende, se acumulan los problemas de aplicación a nuevos contextos. Las modificaciones ad hoc y las adiciones sobre la marcha merman la claridad y elevan innecesariamente la complejidad, resultando una pérdida de eficiencia; de manera que se vuelve crecientemente difícil hacer modificaciones o adiciones y, a la vez, mantener la coherencia (ibid.). Esto constituye un incentivo para empezar de nuevo, sobre todo porque la arquitectura existente impone límites a cualquier innovación específica o incremental que pueda introducirse pasado cierto punto. Así aumenta la presión para romper dicha arquitectura y dar paso a un cambio radical (Nooteboom, op. cit.:183).

Sin embargo, no habría que olvidar que una combinación radicalmente nueva no es fácil de identificar como oportunidad, ya que no tiene sentido en el marco de la práctica existente. Aquí resulta clave la distinción entre invención e innovación, en tanto el proceso de generalización, es decir, la transferencia de la práctica a nuevos contextos, es fuente de invenciones, pero la rutina nulifica o limita su aprovechamiento; es solamente cuando la arquitectura se ve amenazada porque hay mayor receptibilidad a las nuevas ideas o combinaciones.

El siguiente paso consiste en ubicar las revoluciones tecnológicas dentro de la transformación general del sistema capitalista. Esto nos permitirá distinguir la continuidad de las sucesivas eras tecnológicas y lograr una primera aproximación tentativa a la especificidad de la etapa actual, que permita abrir la discusión más amplia del cambio histórico. ${ }^{24}$

\section{Sucesión y especificidad de las eras o revoluciones tecnológicas}

A partir de la revolución industrial, se acelera el desarrollo de las fuerzas productivas del capitalismo, en el cual un avance tecnológico u organizativo central plantea nuevos requerimientos que se arrastran por un tiempo para dar lugar a un nuevo brote brusco, el cual hemos definido como revolución tecnológica. La continuidad está determinada, por un

24 Esa ubicación histórica se basa en los clásicos: Mantoux (1962:194 201, 224 237 y 306 314) y Landes (1969:211 230); autores modernos como Mokyr (2002:29 56; 1990:239 269), Freeman Louca (2001:222 239), y la propuesta de interpretación de Tylecote (1991:36 52).

\section{Desaarrollo}


lado, por el creciente papel del conocimiento mediante la ampliación de la base epistemológica de la tecnología y, por otro lado, cambios inducidos en la organización social, planteados por el propio desarrollo productivo. ${ }^{25}$ Marx capta la continuidad y discontinuidad del progreso tecnológico al plantear la unidad del sistema de máquinas (Marx, 1946, t. I:303). Cada uno de sus tres componentes —máquina herramienta, máquina motriz y máquina de transmisión - experimentan, en sucesión, cambios revolucionarios que imponen transformaciones posteriores en los otros componentes. Esto es lo que tomaremos como base para encontrar la lógica que relaciona una revolución con otra y, por ende, la especificidad de las mismas, en particular de la última (véase Cuadro 1).

Freeman y Louca (2001:139-143) y Pérez (2002:11) hablan de cinco eras o revoluciones tecnológicas, cuyas características se resumen en el Cuadro $1 .^{26}$ La difusión de una revolución tecnológica conduce a la reestructuración de toda la economía, a partir de la modernización de la infraestructura, prácticas óptimas, productos, perfiles de capacitación, patrones de localización de la producción y uso intensivo del insumo clave (Freeman y Louca, op. cit.:147; Pérez, 2002:13-15), es decir, a un nuevo patrón industrial (Dabat y Ordóñez, en prensa:15-16).

Hay consenso entre los especialistas de que los tres elementos fundamentales de la primera era tecnológica o era industrial —algodón, energía hidráulica e hierro— se reforzaron mutuamente para elevar la capacidad de producción industrial en Inglaterra a partir de 1780. El impulso inicial derivó del uso creciente del agua como medio de transporte y

25 El papel del conocimiento como fundamento de la base epistemológica de la tecnología se argumenta ampliamente en Mokyr (2002). Este autor plantea que la cuestión central no es por qué tuvo lugar la revolución industrial, sino por qué tuvo continuidad más allá de 1820; antes hubo constelaciones de macroinvenciones, sobre todo en el siglo xv, con el surgimiento de la tipografía, la primera metalurgia del hierro y los avances en la tecnología de la navegación. No obstante, añade, esas minirrevoluciones industriales se disolvieron antes de que la economía despegara. En otras palabras, señala Mokyr, antes de la revolución industrial la economía estaba sujeta a una retroalimentación negativa, en el sentido de que cada episodio de crecimiento chocaba contra resistencias u obstrucciones, mediadas por largos periodos de estancamiento o declinación. El mecanismo de retroalimentación negativa mejor conocido, pero no el único, es la trampa maltusiana y su principal causa estaría en la limitada base epistemológica de la tecnología. En la era preindustrial, tanto en Europa como en China, las tecnologías se utilizaban aunque la comprensión de sus principios era muy limitada. Aunque la revolución industrial no se apoyó en un importante avance de la ciencia, sí se produjo en los siglos XVI y XVII un extraordinario avance en la forma en que se genera, procesa y disemina el conocimiento útil. El vehículo fue la Ilustración y su monumental obra, la Encyclopédie, la cual redujo los costos de acceso al conocimiento útil, al clasificar las prácticas artesanales y propiciar su comparabilidad y perfeccionamiento (2002, cap. 2, especialmente pp. 33 35). Es interesante constatar que Marx tenía una formulación semejante al hablar de la prehistoria del desarrollo de la humanidad, superada con el advenimiento del maquinismo.

26 Usaremos, por razones de simplicidad expositiva, los conceptos revolución industrial y tecnológica como sinónimos, pero una discusión de fondo exigiría diferenciarlos. Revolución industrial, en el sentido clásico, constituye el proceso del cual emana un nuevo patrón industrial (nuevas industrias, productos, procesos), en tanto que revolución tecnológica, como fenómeno previo, se refiere básicamente a la constitución de una nueva práctica óptima. 
Cuadro 1

Eras industriales o revoluciones tecnológicas

\begin{tabular}{llll}
\hline \multicolumn{1}{c}{ Acontecimiento } & \multicolumn{1}{c}{ País } & \multicolumn{1}{c}{ Innovación } & $A_{\tilde{n} o^{1}}$ \\
\hline I. Revolución Industrial & Inglaterra & $\begin{array}{l}\text { Lanzadera de Arkwright } \\
\text { en Cromford }\end{array}$ & 1771 \\
II. Era del vapor y del ferrocarril & $\begin{array}{l}\text { Inglaterra pasando a } \\
\text { Estados Unidos }\end{array}$ & Ferrocarril & 1829 \\
& $\begin{array}{l}\text { Estados Unidos y Alemania } \\
\text { Bessemer en Pittsburg }\end{array}$ & Carnegie con tecnología & 1875 \\
III. Era del acero y la electricidad & $\begin{array}{l}\text { Estados Unidos seguido de } \\
\text { IV. Producción de mas }\end{array}$ & $\begin{array}{l}\text { Plemania pasando a Europa } \\
\text { Esimer Modelo T, Detroit }\end{array}$ & 1908 \\
V. Era de la información & $\begin{array}{l}\text { Estados Unidos pasando } \\
\text { a Europa y Asia }\end{array}$ & $\begin{array}{l}\text { Procesador INTEL, } \\
\text { California }\end{array}$ & 1971 \\
\hline
\end{tabular}

Fuente: Pérez (2002:11).

1 Año de detonación o Big Bang.

fuente energética; como resultado de ello, el sector más receptivo — textiles de algodóncreció rápidamente. El aumento de la producción de textiles de algodón se basó, en primer lugar, en la maquinización de la hilatura con la lanzadera mecánica de Arkwrigh. Pero esta primera producción fabril presentaba dos problemas: $a$ ) las máquinas fabricadas con hierro no forjado era quebradizas; $b$ ) las fuentes hidráulicas estaban territorialmente muy dispersas, lo cual obligaba a desconcentrar la industria. El primer punto se resolvió relativamente pronto con el uso del hierro forjado, mediante el método Puddling, cuya producción se aceleró en 1790. El uso generalizado del hierro forjado transformó la industria de la maquinaria. Sin embargo, las limitaciones energéticas persistieron porque la máquina de vapor de Watt tuvo un ritmo de difusión muy lento, debido a problemas técnicos no resueltos.

La construcción de la máquina de vapor de alta presión a principios del siglo XIX constituyó el pivote de la segunda revolución industrial. Esa innovación fue posible por los avances precedentes en la producción de máquinas herramientas, centrados en el abaratamiento del hierro forjado. También hubo una inducción de demanda creada por las necesidades energéticas y de transporte derivadas del crecimiento de la industria textil. En 1830, el arribo del transporte ferroviario, con la inauguración de la línea LiverpoolManchester, abarató el transporte y el costo de la energía declinó aún más, gracias a la rápida movilización de los stocks de carbón.

La era tecnológica estructurada por el hierro barato y la máquina de vapor acrecentó la presión en el sector de máquinas herramientas, actuando como propulsor hacia la tercera era o revolución tecnológica. La fuerza del hierro esponja y del forjado estaba limitada por impurezas. A medida que el conocimiento de metalurgia se perfeccionaba, se lograron grandes avances en su eliminación, para llegar al acero; es decir, hierro al que se le redujo las impurezas del carbón. Entre 1850-1880, la sucesión de varias innovaciones radicales

\section{Desaarrollo}


del proceso, principalmente el Bessemer, Siemens y Gilchrist-Thomas, permitieron el advenimiento de la era del acero barato de alta calidad. A su vez, el abaratamiento del acero imprimió un nuevo impulso a la industria de la maquinaria.

Los avances en la estructuración del conocimiento útil y la ampliación de su base epistemológica, relacionada principalmente con la ingeniería de máquinas y la metalurgia, crearon un nuevo marco que explica en buena parte los descubrimientos en inducción electromagnética a partir de 1830, del cual surgió la tecnología del dinamo eléctrico hacia 1860-1870, base de la tercera era tecnológica porque abrió la puerta a la generación y transmisión de un nuevo tipo de energía que se conjugaba con los avances en materiales, maquinismo y medios de comunicación logrados hasta entonces. Pero esa tecnología generó nuevos requerimientos desde el punto de vista tanto de la gestión productiva, como de la social, relacionadas con las exigencias de equipo de una complejidad hasta entonces desconocida (mantenimiento, reparación, contabilidad, coordinación, gestión política). Estas necesidades serían la base para una nueva ruptura en el ritmo de las innovaciones, es decir, el salto a la era de la producción de masas, en la cual tomaría el papel dominante la transformación organizativa como gestión y estructura (cuarta era tecnológica).

Se requería una nueva organización por multitud de razones, sobre todo las que emanaban de la ingeniería de máquinas. Ésta había crecido en importancia económica desde mediados del siglo xvIII y se vio fuertemente impulsada por el arribo del acero y la electricidad, pero su papel lo cumplía sobre la base de una organización artesanal, que agudizaba el problema de la limitada intercambiabilidad de partes. La desarticulación del poder artesanal y de los oficios se logró mediante la fórmula de Taylor, al asignar tareas acotadas y especificadas a trabajadores semiespecializados o no especializados adscritos a maquinaria especializada y garantizar así la continuidad a un ritmo creciente por medio del movimiento de componentes. La idea de la línea de ensamble para mover las partes se tomó de la industria química y se añadió el motor eléctrico.

Hacia principios del siglo xx, existían en Estados Unidos las condiciones para la cristalización de la nueva era tecnológica. Ello se dio en 1915, cuando Henry Ford abrió la primera planta de ensamble de automóviles. En la terminología de Freeman y Pérez, la industria automotriz es una rama receptora; proveedoras son la eléctrica y el petróleo. El taylorismo separó las tres funciones de producción: manufactura, diseño y coordinación, que eran indivisibles y difusas en las primeras fábricas. Las grandes firmas entrañan sobre todo grandes problemas de coordinación. El papel de ingenieros y científicos se volvió cotidiano, para dar lugar después al laboratorio. Dentro de esta revolución organizativa inducida por las transformaciones en la ingeniería de máquinas y la tecnología del dinamo eléctrico, había una debilidad que impulsaría la siguiente transformación. Si el fordismo llegó a grado excelso por medio de la maquinización y la capacidad de transferencia lineal, era débil en la capacidad de control, lo cual compensaba utilizando gran cantidad 
de trabajadores especializados que actuaban como elementos de integración y comunicación. Lo que hacía falta era una máquina inteligente.

En consecuencia, los problemas centrales son la retroalimentación e integración, que se configuran como conflictos de información y comunicación. Las limitaciones en ambos atributos colocaron la producción bajo el peso de una fuerte rigidez que afecta el sistema de máquinas, el uso de la fuerza laboral y la producción final. Ante esos requerimientos, el transistor, la célula de la siguiente revolución tecnológica, debe verse como un instrumento básico de comunicación que enlaza, mediante el lenguaje binario al ser humano con las máquinas y a éstas entre sí. Como señala Castells (1999), el transistor hizo posible procesar los impulsos eléctricos a un ritmo más rápido, de un modo binario de interrupción, paso que constituyó el nuevo código de instrucciones.

Los semiconductores y circuitos integrados —es decir, la integración gigantesca de transistores - tiene, en general, dos destinos: $a$ ) se aplica en artefactos o máquinas a las que provee de instrucciones para su funcionamiento, abriendo un nuevo medio de automatización; $b$ ) se emplea como parte central de la unidad de procesamiento de las computadoras que, de este modo, se convierten en instrumentos fundamentales para el manejo de información y la comunicación. La programación electrónica de maquinaria y de las computadoras rompió la rigidez de la producción de masas, reconfigurando la relación entre los medios de producción, los materiales y productos y la fuerza de trabajo.

Antes del advenimiento de la computadora electrónico-digital, los grandes requerimientos de integración, coordinación y comunicación derivados de la confluencia del dinamo eléctrico, el motor de combustión interna, la ingeniería pesada y la cadena de montaje, se enfrentaron por medios electromecánicos y analógicos (Campbell-Kelly y Aspray, 2004:51). El primer paso al sistema electrónico se dio a fines de 1940, cuando se adoptó la arquitectura Von Neumann, la cual consiste en la lectura de datos mediante un procesador que sigue, en secuencia, un programa de instrucciones apoyada por dispositivos de memoria reprogramable (Flamm, 1988:11). Dicha arquitectura fue la creadora del software, en tanto que modificó el diseño Eckert-Mauchly, basado en el propio hardware para efectuar sus operaciones (Mowery, 1999:143); el principio de almacenar las instrucciones en una memoria que podía modificarse es la base del concepto actual de software (ibid.).

La confluencia entre la electrónica y las telecomunicaciones se profundizó a partir de los sesenta, con la aparición del primer conmutador electrónico en Estados Unidos. El uso de la digitación se dio a partir de 1965, con la introducción del pulse code modulation de ATT (Castells, op. cit.:72). El paso definitivo para la integración de los elementos dispersos de la nueva tecnología parece corresponder a los setenta, con la invención del microprocesador, la computadora personal y la individualización del software como industria independiente (Castells, op. cit.:70 y 73). La concreción del proceso de integración de distintas innovaciones es la tecnología genérica, la cual se plasma, en la era actual, en la computadora

\section{Desaarrollo}


microelectrónica-digital, para usar la denominación propuesta por David (2002:68-77;

Carlsson, 2004). Los efectos de esta tecnología genérica pueden clasificarse así:

a) transformación profunda de las actividades productivas, automatización de procesos e integración en la línea de diseño, manufactura, inventarios, ventas y administración, entre otras.

b) en el marco de la integración de actividades, el diseño se convierte en la actividad fundamental por su capacidad para transformar productos y procesos de manera cada vez más acelerada y ampliada;

c) incremento de la capacidad humana para generar valor y riqueza mediante efectos cuantitativos (aumento de productividad) y cualitativos (calidad y variedad), los cuales devuelven al trabajo su condición compleja, mermada con la revolución organizativa y gerencial del fordismo;

d) formación de redes entre múltiples unidades productivas, integrando a proveedores y usuarios para generar grandes economías de abastecimiento;

e) sustentación de la cadena de actividades productivas en enormes bases de datos y medios cada vez más avanzados de procesamiento de información, gracias a los cuales se integran producción y circulación.

La especificidad de la nueva etapa histórica:

conocimiento e información

La sucesión de las innovaciones que hemos analizado sugiere que la especificidad histórica de la era actual radicaría en una relación crecientemente compleja entre conocimiento útil e información, como soportes de la reproducción social. ${ }^{27}$ Los grandes requerimientos de integración, coordinación y comunicación que crecieron aceleradamente desde principios del siglo xx, colocan a la nueva tecnología genérica - la computadora electrónicodigital - como el eje de articulación del conocimiento y la información.

La percepción de este cambio histórico se expresa en la formulación de dos conceptos, en cierto modo complementarios, los cuales intentan abarcar los aspectos centrales de dicha transformación: economía del conocimiento e informacionalismo. ${ }^{28} \mathrm{~A}$ continuación,

27 Tomaremos el concepto de David y Foray (1995:26 27), en el cual el conocimiento es una facultad que permite a su poseedor actuar intelectual y físicamente; la información consiste en datos estructurados que permanecen ociosos hasta que alguien con los conocimientos suficientes los interpreta y procesa. La relación entre ambos es crucial, ya que existe un pasaje permanente de uno a otro: el conocimiento se transforma en información mediante la codificación, es decir, la transcripción del conocimiento en representaciones simbólicas susceptibles de difundirse y almacenarse (ibid.). La codificación da lugar a nuevas posibilidades cognoscitivas ya que, previo aprendizaje, pueden conducir a la creación de nuevos objetos de conocimiento (ibid.:27).

28 Tomamos el concepto de Castells (op. cit.:43), quien lo define como modo de desarrollo actual. El mérito de su propuesta es que hace hincapié en la fuente de crecimiento de la productividad, pero no 
discutiremos su contenido para tratar de interrelacionarlos e integrarlos en la concepción más amplia del desarrollo actual.

Como dice Foray, la noción de economía del conocimiento pretende introducir la idea de una ruptura en los procesos de crecimiento y en las formas de organización económica, representadas en el papel que adquieren los llamados activos intangibles. ${ }^{29}$ En Estados Unidos, el valor corriente de los activos intangibles de capital empezó a sobrepasar a los de capital tangible (infraestructura, equipos, inventarios y recursos naturales) a fines de los sesenta (op. cit.:22). Sin embargo, el cambio en la composición de los activos es sólo la expresión de un cambio cualitativo en el papel del conocimiento, que exigiría referirse, siguiendo a Mokyr (op. cit.:29-43), a dos factores cruciales: las bases epistemológicas y los costos de acceso. Lo primero se refiere a la comprensión de los fundamentos de las innovaciones, es decir, el saber por qué en comparación al saber cómo. Lo segundo se refiere al grado de difusión de ese conocimiento como condición del progreso social.

Sabemos que es a partir de la revolución industrial cuando se empieza a superar la estrechez epistemológica de la tecnología y quedan atrás los mecanismos de retroalimentación negativa del crecimiento económico. A lo largo del siglo XIX, aunque aumentó la importancia de la ciencia en la innovación tecnológica subsistió un importante desfase entre el por qué y el cómo, notorio aun en el caso del dinamo eléctrico. ${ }^{30}$ Es únicamente a partir de principios del siglo xx cuando tiende a haber una correspondencia entre las dos estructuras, gracias al conocimiento acumulado pero también a una revolución en la organización de la ciencia aplicada, centrada en el perfeccionamiento del laboratorio de investigación y desarrollo, la universalización de la educación y el apoyo público a la ciencia básica. La correspondencia entre el por qué y el cómo cristaliza en la revolución de la física cuántica que conduce la invención del transistor en los años cuarenta (Riordan y Hoddeson, 1997:83-85).

El advenimiento de los semiconductores, como expresión de la profundización y ampliación cualitativas de las bases epistemológicas de la tecnología, se complementó con una reducción dramática de los costos de acceso debido a la capacidad lograda para codificar el conocimiento y convertirlo en información. La creación de la tecnología genérica

logra diferenciar entre conocimiento con información, para lo cual se requiere la línea de análisis de David y Foray (1995).

29 Es decir, la inversión orientada a la producción y diseminación de conocimiento (capacitación, educación I\&D, información y coordinación) y la inversión orientada a sostener la capacidad humana (gastos en salud). (Foray, 2000:22).

30 "La base epistemológica del arco voltaico estaba incompleta aun a finales del siglo xIX, en tanto los científicos estaban divididos entre hipótesis químicas y antiquímicas del funcionamiento del generador. Nelson y Rosenberg (1973:7 8) destacan que Edison observó el flujo de corriente entre el filamento incandescente y el alambre de su lámpara sin percatarse de que estaba observando el movimiento de los electrones, la existencia del cual fue postulada veinte años después" (Mokyr, 2002:93).

\section{Desaarrollo}


de la computadora electrónico-digital explica la reducción dramática de los costos de acceso y la conversión masiva de conocimiento tecnológico a bien público (David y Foray, 1995:27). Así estructurada, la economía del conocimiento tiende a gobernar la reproducción social en las economías más desarrolladas a partir de los años ochenta, ${ }^{31}$ generando los siguientes efectos económicos:

a) se acelera el crecimiento de la productividad principalmente en el procesamiento, almacenamiento e intercambio de información, cruciales en un grupo muy amplio de sectores económicos;

b) surgen nuevos bienes y servicios;

c) se adopta un modelo organizativo de las relaciones de propiedad centrado en las posibilidades de extraer rentas económicas del manejo de la información.

La economía del conocimiento se sustenta en un tipo de tecnología que revoluciona la capacidad para procesar la información, pero su racionalidad económica conduce a un nuevo modelo organizativo. El informacionalismo representa, en consecuencia, ese nuevo modelo organizativo adoptado para extraer rentas económicas de las nuevas posibilidades en el manejo de la información. Lo anterior supone, naturalmente, una conversión masiva de conocimiento a información ${ }^{32}$ y el uso de ésta como insumo productivo gracias a lo cual se incrementa explosivamente el valor de uso de los bienes (mayor calidad, variedad y sucesión acelerada de modelos) y, por ende, su valor. De lo anterior se desprende que el informacionalismo es un proceso acotado por las relaciones de propiedad capitalista y estructurado a partir de elementos tecnológicos y de transformaciones en los derechos de propiedad. Consiste en el uso capitalista del conocimiento a partir del procesamiento de la información como principal fuente del incremento de la productividad. ${ }^{33}$

Si la estructuración de la economía del conocimiento y su interfase con el informacionalismo le confiere gran importancia a los sistemas en el marco de una implacable progresión de la automatización, ${ }^{34}$ la clave para valorar el capital depende de la nueva función

31 Con la tecnología electrónico informática, la economía del conocimiento encontró su base material (Foray, op. cit.:27).

32 "La transformación del conocimiento en información es una condición necesaria para convertirlo en mercancía". Ello explica porqué el mercado de conocimiento es muy delgado, es decir, constituye un monopolio bilateral, en el cual el comprador y el vendedor asignan al bien un valor discrepante (David y Foray, 1995:26 y 31). Dado que la valoración se fundamenta en la información, es prefe rible hablar de capitalismo informático que de capitalismo cognoscitivo.

33 Paradójicamente, como explican Cowan, Foray y David (2000:242), en tanto esta nueva organiza ción productiva se basa en una elevada codificación del conocimiento como condición para la valoración, pone en peligro su capacidad de rendir plusvalor, ya que aproxima el conocimiento a bien público no rivalizable. Veremos ese punto en la parte final de este apartado.

34 La automatización sigue un ritmo implacable, pero su velocidad ha sido sobreestimada. El principal obstáculo son los problemas de comunicación entre sistemas y autómatas, lo cual exige enormes

\section{Desaarrollo}


del trabajo humano. Aunque hay un incremento extraordinario de lo que Marx llamó composición técnica, el trabajo humano se potencia como trabajo complejo a partir de tres actividades centrales:

a) la programación como creador del lenguaje de instrucción para las computadoras electrónico-digitales, principalmente en la modalidad de aplicaciones para el desarrollo de software (software para crear software);

b) la concepción del producto o proceso mediante el diseño y su cristalización como prototipo comercializable (Ordóñez, 2004);

c) como eje de la coordinación global del proceso productivo, interconecta sus diferentes estructuras espaciales para dar continuidad a la alta valoración capitalista.

La significación de estas tres actividades es enorme en varios sentidos, pero destaca la tercera, por sus repercusiones ulteriores en el proceso de valoración. Al aumentar el poder de interconexión y coordinación a distancia, se logra, mediante las cadenas globales de producción, acceso a enormes reservas de fuerza laboral de diferente grado de calificación y, por ende, de capacidad para rendir trabajo complejo. El acceso a estas reservas mundiales es, en sí, una fuente de ganancias extraordinarias, ya que los costos de reproducción de esa fuerza de trabajo son sustancialmente más bajos que la media de las economías desarrolladas, en las cuales están implantadas las principales estructuras de la economía del conocimiento.

La contradicción en que se desenvuelve el capitalismo informático es que si la valoración se sustenta en una transformación creciente del conocimiento en información, esa misma transformación lo acerca a la condición de bien público, que corroe la apropiación privada (Foray, op. cit.:113 y ss; David y Foray, op. cit.:27). De acuerdo con diversos autores citados, la respuesta radicaría en la imposición de estrictos derechos de propiedad que restrinjan la difusión de la información y eleven los costos de acceso. ${ }^{35}$ Sin embargo,

capacidades de programación o producción de software que rebasan las ganancias derivadas de la eliminación de fuerza de trabajo. Los cuellos de botella en las actividades de programación persisten en la actualidad y, entrado el siglo xxi, preserva un carácter artesanal, que recuerda a la ingeniería de máquinas a fines del siglo xIx. Por otro lado, la globalización, al abrir un nuevo caudal de fuerza laboral, modula el ritmo de la automatización.

35 Moulier Boutang (s/f, p. 6) sostiene la tesis de que la revolución tecnológica se sustenta en un refor zamiento de los derechos de propiedad, los cuales equipara con los cercados de la revolución industrial. Pero la investigación histórica de la escuela de Stanford (Rosenberg, Mowery, entre otros) refuerza la hipótesis del ciclo de vida en la constitución de los derechos de propiedad, es decir, una sucesión de debilitamiento y fortalecimiento. Para ampliar la base epistemológica de la tecnología se requirió un enorme esfuerzo de investigación básica, la cual fue financiada por el gobierno estadounidense en beneficio de toda la clase capitalista y, por ende, implicó la difusión de los derechos de propiedad. Posteriormente, como explican Mowery y Rosenberg (1999:36 37), ese esfuerzo se debilitó después de los setenta, tanto por restricciones presupuestarias como por ser menos necesario para mantener

\section{DeSaarrollo}


la misma estructuración histórica de la economía del conocimiento ha dependido del abatimiento de los costos de acceso al conocimiento. Lo anterior sugiere que los derechos de propiedad no juegan un papel uniforme a lo largo del ciclo de difusión del nuevo paradigma, sino que se definen socialmente a partir del ciclo de vida tecnológico. Siguiendo la terminología de Afuah y Utterback (1977:185), en la fase fluida del ciclo tecnológico los derechos de propiedad se difunden, es decir, se amplía el acceso al conocimiento tecnológico; en contraste, una vez constituido el diseño dominante, empiezan a aparecer fuertes tendencias a la centralización de los derechos de propiedad, es decir, a elevar los costos de acceso. $^{36}$

\section{Conclusión}

La determinación de la especificidad histórica de la etapa actual del desarrollo del capitalismo mundial constituye una tarea intelectual crucial, tanto para entender las fuerzas que gobiernan la reproducción social como para formular estrategias acordes con las nuevas exigencias históricas. El reconocimiento de esta prioridad forma parte de la constitución de un nuevo pensamiento económico-social, que gradualmente tiende a ocupar el vacío dejado por la ortodoxia neoclásica, enfrentada de manera regular a fracturas internas de carácter epistemológico.

En el marco de estas nuevas posibilidades para orientar la discusión teórica y la investigación, el presente artículo integró diversas aportaciones al estudio del cambio histórico, siguiendo la propuesta formulada por Hodgson. El hilo conductor fue la recuperación de elementos de la teoría de Marx y Veblen, que sirven de base al replanteamiento actual del cambio histórico, en el que participan varias escuelas y corrientes (institucionalistas, neoschumpeterianos y regulacionistas). El primer objetivo fue partir de esas aportaciones a fin de llegar a una nueva propuesta para enmarcar la especificidad histórica del capitalismo. Ese teorización tentativa, de la cual surgieron varias hipótesis, se ubicó en el marco del análisis histórico constituido por el examen de los aspectos más sobresalientes de la

la continuidad de las actividades de innovación en la industria. Parece ser que a partir de los ochenta se abre el periodo de reforzamiento o centralización de los derechos de propiedad, como lo sugieren diversos eventos entre los que sobresale el desenlace de los juicios antitrust contra Microsoft.

36 Existen tres ejemplos históricos de difusión de los derechos de propiedad, para emplear el término utilizado por Mowery y Rosenberg. En abril de 1952, laboratorios Bell organizó un simposio en el que reveló la tecnología de punto de contacto para el transistor, gracias a lo cual llegó a un acuerdo para evitar un juicio antitrust (op. cit.:125). El documento en el cual Von Neumann expuso el principio de la memoria reprogramable de la computadora, elemento central de su diseño arquitectónico hasta nuestros días, circuló libremente a fines de los años cuarenta (Mowery, 1999:143 infra). Final mente, en 1970, IBM desató el sistema hardware del software en el modelo 370 y posteriores, aban donó su estrategia de incompatibilidad y procedió a traspasar rentas económicas a sus seguidores (Bresnahan y Malerba, 1999:96); lo anterior condujo posteriormente, en la era de la PC, a la decisión de obtener componentes para sus microcomputadoras de fuentes externas, principalmente de Intel y Microsoft, sin forzarlos a restringir la venta de esos componentes a terceros (Mowery, op. cit::144). 
sucesión de las eras tecnológicas. El objetivo fue esbozar una heurística para interpretar la interconexión de una revolución tecnológica a otra.

Al trasladar ese análisis a la etapa actual, se formula la hipótesis de que la especificidad radica en una relación compleja entre conocimiento e información, posibilitada por la ampliación de la base epistemológica del conocimiento y, complementariamente con ello, por el advenimiento de la computadora electrónica-digital como tecnología genérica. Los temas que se abren a partir de este planteamiento son enormes y habría sido imposible pasar, en este trabajo, más allá de un esbozo sesgado hacia ciertos ángulos. No obstante, en aras de reforzar algunas precisiones, sería pertinente subrayar que en tanto el concepto economía del conocimiento tiende a expresar el valor de uso social de la revolución de las fuerzas productivas, el informacionalismo remite a la estructuración de esas capacidades a partir de las relaciones de propiedad. Ambas estructuras tienden a complementarse y a apoyarse mutuamente, ya que el dinamismo tecnológico se explica desde las condiciones de apropiabilidad que norman la actuación de los agentes empresariales. Sin embargo, a medida que se difunde la nueva tecnología y se articula un nuevo patrón industrial, el conflicto entre la normatividad de la tecnología de propietario y la constitución de comunidades en torno al conocimiento como bien público se hace más encarnizado. La respuesta capitalista a la creciente conversión de conocimiento en bien público consiste en endurecer los derechos de propiedad y erigir nuevas barreras a la difusión del conocimiento. Esta contradicción pone al descubierto la pugna perenne del capitalismo por imponer su lógica (basada en el valor) a la reproducción social y a la satisfacción de las necesidades humanas. Esta confrontación, lejos de haberse atenuado por la extraordinaria renovación de las fuerzas productivas, reaparece ahora con un nuevo ropaje histórico.

En el marco del estudio y del debate, la economía política crítica debiera adoptar el compromiso intelectual derivado del cambio histórico, abandonar los enfoques mecanicistas y rígidos a favor de formulaciones abiertas, centradas en la necesidad de combinar el análisis histórico con la investigación empírica. De manera normativa, el compromiso debe situarse del lado de la progresividad histórica como condición necesaria para lograr la integración de las grandes mayorías.

Considerando la fuerza de la transformación del conocimiento en bien público, la hegemonía que se requiere para estabilizar la reproducción social — y dar durabilidad y estabilidad a las trayectorias de crecimiento - parece reclamar una transformación profunda de las relaciones de propiedad, equivalente a la extraordinaria transformación de las fuerzas productivas. En esta perspectiva, la noción de bloque histórico de Gramsci conserva todo su valor para comprender sistémicamente este complejo proceso. 


\section{Bibliografía}

Afuah, Allan y James Utterback, "Responding to Structural Industry Changes: A Technological Evolution Perspective", en Industrial and Corporate Change, núm. 1, 1997, pp. 183-201.

Bresnahan, T. y F. Malerba, "Industrial Dynamics and the Evolution of Firm's and Nations' Competitive Capabilities in the World Computer Industry”, en D. Mowery y R. Nelson (editores), Sources of Industrial Leadership. Studies of Seven Industries, Cambridge, Cambridge University Press, 1999.

Boutang Moulier, Yann, Los nuevos cercamientos: nuevas tecnologías de la información y de la comunicación, o la revolución rampante de los derechos de propiedad, París, Universidad de París-La Sorbona, inédito.

Boyer, Robert, "The Diversity and Future of Capitalism: A Regulationist Analysis", en G. Hodgson, M. Itoh y N. Yakokawa (editores), Capitalism in Evolution: Global Contention. East and West, Londres, Edwar Elgar, 2001.

- "Technical Change and the Theory of Regulation", en G. Dosi, et al., Technical Change and Economic Theory, Londes, Pinter Publisher, 1988.

, "La crisis en una perspectiva histórica. Algunas reflexiones a partir de un análisis a largo plazo del capitalismo francés", en R. Conde (compilador), La crisis actual y los modos de regulación del capitalismo, en Cuadernos Universitarios, núm. 8, México, UAm-Iztapalapa, 1984.

Brenner, Robert y M. Glick, "The Regulation School and the West's Economic Impasse", en New Left Review, núm. 188, julio/agosto de 1991.

Campbell-Kelly y W. Aspray, Computer. A History of the Information Machine, Boulder, Westview Press, 2004.

Carlsson, Bo, "The Digital Economy: What is New and What is Not?", en Structural Change and Economic Dynamics, núm. 15, 2004, pp. 245-264.

Castells, Manuel, La era de la información, vol. I, México, Siglo XXI, 1999.

Clark, John; Christopher Freeman y L. Soete, "Long Waves, Inventions and Innovations", en C. Freeman (coordinador), Long Waves in the World Economy, Londres, Butterworths, 1983.

Clark, N. y C. Juma, "Evolutionary Theories in Economic Thought", en G. Dosi et al., Technical Change and Economic Theory, Londres, Pinter Publisher, 1988.

Cowan, Robin, Paul A. David and Dominique Foray, "The Explicit Economics of Knowledge Codification and Tacitness", en Industrial and Corporate Change, vol. 9, núm. 2, 2000. y Dominique Foray, "The Economics of Codification and the Diffusion of Knowledge", en Industrial and Corporate Change, vol. 6, núm. 3, 1997, pp. 595-622.

Dabat,Alejandro, Capitalismo mundial y capitalismos nacionales, México, Fondo de Cultura Económica, 1994.

__ El mundo y las naciones, México, CRIMUNAM, 1993.

- "Globalización, capitalismo actual y nueva configuración espacial del mundo", en J. Basave, A. Dabat et al. (coordinadores), Globalización, inserción de México y alternativas incluyentes, México, IIEC/CRIM/UAm/Porrúa, 2002.

- y M.A. Rivera, "Nuevo ciclo industrial mundial e inserción internacional de países en desarrollo", en A. Dabat, M. Rivera y J. Wilkie (coordinadores), Globalización y cambio tecnológico. México en el nuevo ciclo industrial mundial, México, Universidad de Guadalajara/UnAM UCLA-Program on Mexico/ Juan Pablos, 2004.

David, Paul A., "Understanding the Digital Technology's Evolution and the Path of Measured Productivity Growth: Present and Future in the Mirror of the Past", en Erik Bryjolfsson y Brian Kahin (editores), Understanding the Digital Economy. Data, Tools and Research, Cambirdge, The MIT Press, 2002.

y D. Foray, "Accessing and Expanding the Science and Technology Knowledge Base", en STI Review, núm 16, 1995, pp. 13-65.

Foray Dominique, Economics of Knowledge, Cambridge, The MIT Press, 2000.

Freeman, Christopher, "Prometheus Unbound", en Futures, octubre de 1984.

, "Technical Innovation and Long Waves in the World Economic Development", en Futures vol. 13, núm. 4, 1981.

y F. Louca, As Time Goes By. From de Industrial Revolutions to the Information Revolution, Oxford, Oxford University Press, 2001.

- y Carlota Pérez, "Structural Crises of Adjustment: Business Cycles and Investment Behaviour", en G. Dosi, R. Nelson, G. Silverberg, y L. Soete (editores), Technical Change and Economic Theory, Londres, Pinter Publishers, 1988.

Glasner, David, Business Cycles and Depressions. An Encyclopedia, Nueva York, Garland Publishing, 1997.

Harvey, David, Los límites del capitalismo y la teoría marxista, México, Fondo de Cultura Económica, 1990. 
-, The Condition of Postmodernity. An Enquiry into de Origins of Cultural Change, Cambridge, Blackwell, 1990a.

Helpman, E. y M. Trajtenberg, "Diffusion of General Purpose Technology", en E. Helpman (editor), General Purpose Technologies and Economic Growth, Cambridge, The MITT Press, 1998.

Hodgson, Geoffrey, How Economics Forgot History. The Problem of Historical Specificity in Social Science, Londres, Routledge, 2001.

, "On the Evolution of Thorstein Veblen's Evolutionary Economics", en Cambridge Journal of Economics, núm. 22, 1998, pp. 415-431.

- Evolution and Institutions. On Evolutionary Economics and the Evolution of Economics, Cheltenham, Edward Elgar, 1999.

Kenneth, Flamm, Creating the Computer, Washington, The Brookings Institution.

Kondratieff, N., "The Long Waves in Economic Life", en Review of Economics and Statistics, vol. XVII, núm. 6, noviembre 1925 , reeditado en C. Freeman (editor), Long Wave Theory, Chentenham, Elgar Reference Collection, 1996.

- Los ciclos largos de la coyuntura económica, México, IIEC-UNAM, 1992 (1926).

Kuhn, Thomas S., La estructura de las revoluciones científicas, México,FondodeCulturaEconómica, 1971.

Landes, David, The Unbound Prometheus. Technological Change and Industrial Development in Western Europe from 1750 to the Present, Londres, Cambridge University Press, 1969.

Langlois, Richard y E. Steinmueller, "The Evolution of Competitive Advantage in the Worldwide Semiconductor Industry, 1947-1996" en D. Mowery y R. Nelson (editores), Sources of Industrial Leadership. Studies of Seven Industries, Cambridge University Press, 1999.

Grasmci, Antonio, Notas sobre Maquiavelo, sobre la política y sobre el estado moderno, Buenos Aires, Nueva Visión, 1972.

Mantoux, Paul, La revolución industrial en el siglo XVIII, Madrid, Aguilar, 1962.

Marglin, Stephen, "Lesson from the Golden Age", en Stephen Marglin y Juliet Schor (editores), The Golden Age of Capitalism. Reinterpreting the Postwar Experience, Oxford, Calredon Press, 1990.

March, James, "Exploration and Exploitation in Organizational Learning", en M. Cohen y L. Sproull (editores), Organizational Learning, Thousand Oaks, Sage Publications, 1996.

Marx, Carlos, La ideología alemana, México, Grijalbo, 1987.

, El Capital. Crítica de la economía política,

México, Fondo de Cultura Económica, 1946.

- "Prólogo de la Contribución a la crítica a la economía política", en C. Marx y F. Engels,
Obras escogidas, t. 1, Moscú, Progreso, y México, Ediciones de Cultura Popular, s/f.

y F. Engels, "Manifiesto del Partido Comunista”, en C. Marx y F. Engels, Obras escogidas, t. 1, Moscú, Progreso, y México, Ediciones de Cultura Popular, s/f.

Mensch, G., Stalemate in Technology: Innovations Overcome Depression, Cambridge, Cambridge University Press, 1979.

Mokyr, Joel, The Gift of Athena. Historical Origins of the Knowledge Economy, Princeton, Princeton University Press, 2002.

_ - The Lever of Riches. Technological Creativity and Economic Progress, Nueva York, Oxford University Press.

Morowski, P., More Heat than Light: Economics as Social Physics, Physics as Nature Economics, Cambridge, Cambridge University Press, 1989.

Mowery, D., "The Computer Software Industry", en D. Mowery y R. Nelson (editores), Sources of Industrial Leadership. Studies of Seven Industries, Cambridge, Cambridge University Press, 1999.

$-\mathrm{y}$ N. Rosenberg, Technology and the Pursuit of Economic Growth, Cambridge, Cambridge University Press, 1989.

, "The Us National Innovation System. A Comparative Analysis", en Richard Nelson (editor), National Innovation System, Oxford, Universidad de Oxford, 1993.

Nooteboom, Bart, Learning and Innovation in organization and Economies, Oxford, Oxford University Press, 2000.

Ordóñez Sergio, "La nueva fase del desarrollo y el capitalismo del conocimiento: elementos teóricos", en Comercio Exterior, vol. 24, núm. 1 , enero de 2004.

Pérez, Carlota, Technical Revolutions and Financial Capital, Cheltenham, Edward Elgar, 2002.

_ - "Cambio tecnológico y oportunidades de desarrollo como blanco móvil", en Revista de la CEPAL, diciembre de 2001.

__ "Structural Change and Assimilation of New Technologies in the Economic and Social System", en Futures, octubre de 1983.

Riordan, Michael y Lillian Hoddeson, Cristal Fire. The Invention of the Transistor and the Birth of the Informational Age, Nueva York, W. W. Norton \& Company, 1997.

Rivera Ríos, Miguel Á., Capitalismo informático, cambio tecnológico y desarrollo nacional, México, Juan Pablos, en prensa.

, "Cambio tecnológico y reproducción social. El perfil de una nueva teoría económica", ponencia, Taller sobre economía del conocimiento, Universidad de París 1-La Sorbona, 17 al 23 de septiembre de 2004 .

\section{Desaarrollo}




\section{MIGUEL ÁNGEL RIVERA RÍOS}

Rutherford, Malcolm, "Veblen's Evolutionary Programme: a Promise Unfulfilled", Cambridge Journal of Economics, núm. 22, 1998, pp. 415-431.

Sandoval, Luis, Introducción a los ciclos largos de la coyuntura económica de N. Kondrátiev, México, IIEC/Unam, 1992.

Schumpeter, Joseph, Business Cycles. A Theoretical, Historical and Statistical Analysis of the Capitalism Process, Filadelfia, Porcupine Press, 1964.

Solomou, Solomos, Phases of Economic Growth, 1850-1973. Kondratieff Waves and Kuznets Swings, Cambridge, Cambridge University Press, 1988.
The Economist, Managing Complexity, Special Report on Software Development, $27 \mathrm{de}$ noviembre de 2004.

Tylecote, A., The Long Wave in the World Economy, Londres, Routledge, 1991.

Veblen, T., The Place of Science in Modern Civilization and Other Essays, New Bruswick, Transaction Publisher, 1961 (1919).

- Teoría de la clase ociosa, Un estudio económico de las instituciones, México, Fondo de Cultura Económica, 1963 (1899).

-, "Why is Economics not an Evolutionary Science?", en Cambridge Journal of Economics, núm. 22, 1998 (1919), pp. 403-414. 